\title{
Effect of an Educational Intervention on the Knowledge about Traumatic Brain Injury Guidelines among Clinical Nurses
}

\author{
Sivakami Subramaniam ${ }^{1}$ Preetham Prabhakar ${ }^{2} \quad$ Umadevi Kanagaraj $^{2} \quad$ Priya Baby, ${ }^{3, \odot}$ \\ ${ }^{1}$ National Institute of Mental Health and Neurosciences, Bengaluru, \\ Karnataka, India \\ 2Kempegowda Institute of Nursing Sciences, Bengaluru, Karnataka, \\ Address for correspondence Priya Baby, MSN, PhD, College of \\ Nursing, National Institute of Mental Health and Neurosciences, \\ India \\ ${ }^{3}$ College of Nursing, National Institute of Mental Health and \\ Neurosciences, Bengaluru, Karnataka, India \\ Bengaluru 560011, Karnataka, India (e-mail: priam19@gmail.com).
}

Indian J Neurotrauma 2022;19:127-131.

\begin{abstract}
Objective Traumatic brain injury (TBI) is a major cause of death and disability throughout the world including developing countries like India. Adherence to guideline based TBI management has proven to decrease death rate and improve neurological outcomes. This study aimed to assess the knowledge of TBI guidelines among clinical nurses caring for TBI patients, and to determine the effect of an educational intervention on their knowledge.

Methods Preexperimental, one group pretest and posttest design was used. A purposive sample of 60 nurses was included in the study. A structured questionnaire was prepared to assess the knowledge of nurses. Educational intervention was given in the form of a planned teaching program that was prepared based on the national TBI guidelines.

Keywords

- traumatic brain injury

- clinical guidelines

- nurses

- knowledge

- educational intervention

Results There was a significant gain in the knowledge scores of the nurses after the educational intervention when compared with the knowledge scores before the intervention $(p=0.001$ ) Nurses who were experienced more than 10 years were found to have significantly higher knowledge score than others with lesser years of experience. Discussion This study demonstrates important knowledge gap about TBI guidelines among clinical nurses. Educational interventions can be effectively used to improve the knowledge about TBI guidelines among nurses.
\end{abstract}

\section{Introduction}

The term traumatic brain injury (TBI) is used primarily to signify craniocerebral trauma, which includes an alteration in consciousness. TBI causes physical, emotional, intellectual, and social challenges for the survivor. ${ }^{1}$

TBI constitutes a major proportion of the global injury burden and there is an increase in its incidence over the published online July 7, 2021
DOI https://doi.org/ 10.1055/s-0041-1729140 ISSN 0973-0508 (c) 2021. Neurotrauma Society of India.

This is an open access article published by Thieme under the terms of the Creative Commons Attribution-NonDerivative-NonCommercial-License, permitting copying and reproduction so long as the original work is given appropriate credit. Contents may not be used for commercial purposes, or adapted, remixed, transformed or built upon. (https://creativecommons.org/licenses/by-nc-nd/4.0/).

Thieme Medical and Scientific Publishers Pvt. Ltd. A-12, 2nd Floor, Sector 2, Noida-201301 UP, India 
recent years. ${ }^{2}$ TBIs are a major public health problem in India, resulting in disabilities and deaths of young and productive people of society. India has the highest rate of traumatic injuries, with an incidence of 1 million trauma-related deaths every year, of which half is caused by TBIs. ${ }^{3}$ In the city of Bangalore alone 10,000 cases are reported in a year resulting in more than 1000 deaths. ${ }^{4}$ One out of six persons who sustain trauma dies in India. In developed countries like the United States, this is 1 out of $200 .^{5}$ This can be reflective of the inadequate coordination and implementation of prehospital and intrahospital care of trauma victims. Ninety-five percent of trauma victims in India do not receive optimal care during the initial 1 hour usually referred to as the "golden hour" period after an injury. ${ }^{5}$

Hospitals should be equipped with laid down clinical protocols for timely care of patients with TBI. Clinical guidelines can potentially improve the quality and process of care. ${ }^{6}$ TBI guidelines are comprehensive and provide an updated review of the rationale for each therapeutic decision. The quality and consistency of clinical care of head injured patients have been found to be enhanced by the availability of clear evidence-based guidelines. ${ }^{7}$ Adherence to guideline-based TBI management protocols can reduce mortality and significantly improve neurologic outcomes. ${ }^{89}$ However, many institutions do not rely on laid-down guidelines and protocols for care of TBI patients. ${ }^{10}$ Nursing professionals are responsible for monitoring and taking care of patient with TBI. Studies have highlighted the need for education and specialty training among nurses to establish best practices in TBI care. ${ }^{11-14}$ Hence, it is vital for nurses to acquire knowledge in TBI guidelines and deliver care based on them.

Training of the nursing staff is crucial in this regard and very often nurse managers are responsible for planning such educational programs for nurses. ${ }^{15}$ Hence, the present study was undertaken to assess the level of knowledge of clinical nurses about care of TBI patients and to determine the effect of an educational intervention in the form of a planned teaching program regarding TBI guidelines ${ }^{8}$ on the knowledge levels of nurses.

\section{Methods}

This was a preexperimental study performed among clinical nurses working in an 800-bedded multispecialty hospital in Bangalore, India. This hospital receives an average of 25 trauma patients every day in the emergency department. It has a dedicated emergency intensive care unit (ICU) and surgical ICU that mostly have in-patient trauma care patients.

\section{Participants}

The participants were selected through a purposive sampling technique. Registered nurses who were working in the emergency department, surgical ICU, or emergency ICU (units where TBI patients are admitted) were included in the study. A total of 150 nurses are posted in these areas. Out of the 150 nurses, nurses who were available during data collection were included. Nurses who did not give informed consent were excluded. Thus, the total sample consisted of 60 clinical nurses.

\section{Measures}

A self-administered questionnaire was used for the study. The questionnaire consisted of two main parts. The first part consisted of eight items related to sociodemographic characteristics of respondents such as age, gender, religion, marital status, professional qualification, and years

Table 1 Subsections of the structured questionnaire and examples of items included in it

\begin{tabular}{|l|l|l|l|}
\hline SL. no & Subsections & No. of items & Examples of item \\
\hline 1 & Concepts of TBI (recall based item) & 16 & $\begin{array}{l}\text { Most common cause of TBI is Absence of safety policies } \\
\text { Rapid urbanization } \\
\text { Motor vehicle collision } \\
\text { Infection }\end{array}$ \\
\hline 2 & $\begin{array}{l}\text { Pre hospital care of TBI guidelines } \\
\text { (application-based item) }\end{array}$ & 7 & $\begin{array}{l}\text { The immediate action performed to open the airway } \\
\text { manually following TBI is Clearing the oral cavity } \\
\text { Suction } \\
\text { Intubation } \\
\text { Jaw thrusta }\end{array}$ \\
\hline 3 & $\begin{array}{l}\text { In hospital care of TBI guidelines } \\
\text { (application-based item) }\end{array}$ & 14 & $\begin{array}{l}\text { Hypotension following acute TBI is managed with } \\
\text { Starting large caliber peripheral line with normal saline } \\
\text { Starting medium caliber peripheral line with dextrose }\end{array}$ \\
\hline 4 & $\begin{array}{l}\text { Galine } \\
\text { Starting small caliber peripheral line with ringer lactate } \\
\text { Starting small caliber peripheral line with digitalis }\end{array}$ \\
\hline (recall-based item) & $\begin{array}{l}\text { The secondary preventive measure of TBI focuses on } \\
\text { Public awareness activities and avoid falls in elderly } \\
\text { Provision and use of safety equipment } \\
\text { Setting safety standards and supervision } \\
\text { Early recognition and prevention of complications }\end{array}$ \\
\hline
\end{tabular}

Abbreviation: TBI, traumatic brain injury

${ }^{a}$ Correct response. 
of clinical experience. Second part of questionnaire consisted of 40 items on knowledge regarding TBI guidelines. There were four subsections in this part, each subsection having items pertaining to different aspects of care. The subsections included basic concepts of TBI, guidelines for prehospital care of TBI, guidelines for in-hospital care of TBI, and guidelines for preventive care of TBI. The details of the subsections and examples of items included in each subsection are mentioned in - Table 1. Each item had four options with one correct answer. A score of one was given for correct response and zero for incorrect response. Whenever nurses did not give response to an item or when two responses were made, a score of zero was given. Thus, the maximum obtainable score was 40 and minimum score was zero. The knowledge level was categorized into three groups: inadequate knowledge ( $\leq 50 \%$ score), moderately adequate knowledge (51-75\% score), and adequate knowledge (>75\% score).

Content validity of the tool was established by obtaining suggestions from the experts including medical surgical nursing specialists, neurosurgeons, and clinical psychologist. The tool was modified based on the suggestions received from experts. Internal consistency of the tool was established by split half method in which the scoring of one half of the tool was compared with the scoring of other half of the tool. This was done in another hospital to avoid contamination of the study sample. The spearman's coefficient of the split half test was 0.85 .

Educational intervention for this study was designed in the form of a planned teaching program. The teaching program was developed based on the guidelines given by Neurotrauma Society of India. ${ }^{7}$ The steps involved in the development of the educational intervention were adoption of TBI guidelines, preparation of appropriate AV aids, and planning for teaching.

The questionnaire was piloted among ten clinical nurses (working in another hospital) to check the practical feasibility of the study like duration of time needed for answering the questions and comprehensibility of questions. Around 45 minutes of time was needed for each nurse to complete the questionnaire. No major changes were made in the questionnaire after the pilot study.

\section{Procedure}

All the nurses who volunteered to participate were gathered for data collection. These included clinical nurses working at the bed side and involved in direct caregiving. In-charge nurses and other nurses holding primarily administrative roles were not included in the study. The purpose of the study, its duration, and nature was explained to the participants. Data were collected in the month of February 2017.

The validated questionnaire was administered (pretest) to the participants. Educational intervention was administered forthenursesfollowingthepretest. Thesameinvestigators(first author and second author) were involved in administration of the questionnaire as well as the educational intervention. The educational intervention included a didactic lecture with power points explaining clinical situations and possible nursing actions in those situations with their rationale. The need for guidelines, various aspects of it, and the practical ways of implementing it in the local setup were presented. Discussion was encouraged, the practical concerns in implementing nursing actions were brought forward with possible solutions, and questions were answered. It lasted for 2 hours.

The posttest session was conducted after 1 week of the educational intervention. The same structured knowledge questionnaire that was used for the pretest was used to collect the posttest data.

\section{Results}

All the nurses who participated in the study were females. Majority of them (43.3\%) were less than 30 years of age. Most of the respondents (76.7\%) were living in a nuclear family. A huge majority of the nurses $(93.3 \%$ ) had completed diploma in nursing and the others were graduates. Most of them (73.3\%) were having total clinical experience less than 10 years. This included not only their experience in caring for TBI patients, but the cumulative years of clinical experience after completion of their professional course in nursing.

Categorization of the pretest and posttest knowledge of the nurses is explained in - Table 2. Majority of them (93.3\%) had inadequate knowledge regarding TBI guidelines before the intervention.

Out of the total score of 40 , the pretest score ranged from 6 to 22 and the mean pretest score of the sample was 14.31 \pm 3.84 . The posttest scores ranged from 28 to 38 with a mean posttest score of $34.78 \pm 2.23$. A paired $t$-test showed that there was a significant difference between the mean pretest and posttest scores of the nurses ( $p=0.001$ ). Subsection-wise change in mean knowledge score is depicted in - Table 3.

The sociodemographic variables were not normally distributed. Hence, the nonparametric, chi-squared test was used to test the association between knowledge and sociodemographic variables. The pretest score of the sample was not found to be associated with any of the sociodemographic variables such as age, marital status, or professional qualification. The relationship between years of experience and knowledge score was found to be significant. ( $\chi 2=10.16, p=0.05$ ).

Table 2 Categorization of pretest and posttest knowledge level of nurses, $n=60$

\begin{tabular}{|l|l|l|}
\hline \multirow{2}{*}{$\begin{array}{l}\text { Knowledge } \\
\text { level }\end{array}$} & Pretest & Posttest \\
\cline { 2 - 3 } & Frequency (\%) & Frequency (\%) \\
\hline $\begin{array}{l}\text { Inadequate } \\
<50 \% \text { score }\end{array}$ & $56(93.3)$ & $\begin{array}{l}0 \\
(00)\end{array}$ \\
\hline $\begin{array}{l}\text { Moderate } \\
50-75 \% \\
\text { score }\end{array}$ & $4(6.7)$ & $\begin{array}{l}4 \\
(6.7)\end{array}$ \\
\hline $\begin{array}{l}\text { Adequate } \\
>75 \% \text { score }\end{array}$ & $0(0)$ & $\begin{array}{l}56 \\
(93.3)\end{array}$ \\
\hline
\end{tabular}


Table 3 Comparison of pretest and posttest knowledge scores, $n=60$

\begin{tabular}{|l|l|l|l|}
\hline \multirow{2}{*}{ Subsections } & Pretest & Posttest & \multirow{2}{*}{$p$-Value } \\
\cline { 2 - 3 } & Mean \pm SD & Mean \pm SD & 0.001 \\
\hline Basic concepts of TBI & $5.43 \pm 1.960$ & $13.41 \pm 1.47$ & 0.001 \\
\hline Guidelines for prehospital care of TBI & $1.88 \pm 1.20$ & $5.70 \pm 0.94$ & 0.001 \\
\hline Guidelines for in-hospital care of TBI & $5.88 \pm 2.21$ & $12.73 \pm 1.08$ & 0.001 \\
\hline Guidelines for preventive care of TBI & $1.11 \pm 0.84$ & $2.93 \pm 0.25$ & 0.001 \\
\hline Total & $14.31 \pm 3.84$ & $34.78 \pm 2.23$ & \\
\hline
\end{tabular}

Abbreviations: TBI, traumatic brain injury; SD, standard deviation.

\section{Discussion}

This study primarily demonstrated the effectiveness of an educational intervention on the knowledge of clinical nurses regarding TBI guidelines. Assessment of the knowledge is essentially the first step in implementing any evidence-based guidelines or protocols in clinical settings. The findings of this study confirm that there is a need for enhancement of the knowledge of nurses pertaining to use of guidelines in TBI care. There was a considerable improvement in knowledge after the educational intervention regarding TBI guidelines among clinical nurses.

The sociodemographic characteristics of the sample were not found to be associated with knowledge levels of the nurses. However, nurses with clinical experience of more than 10 years were found to have better knowledge than nurses with lesser experience. Oyesanya et al have also shown that nurses having more clinical experience have better knowledge regarding TBI care and guidelines. ${ }^{16}$ This can be attributed to the involvement of the nurses in their work. Experienced nurses are always a worthy resource of the TBI care team. Hence, this finding is in congruence with the general understanding that experience in clinical area adds on to ones' knowledge and skill sets.

The mismanagement of TBI patients beginning from the site of event and continuing into the hospital care and rehabilitation has a major role in contributing to the huge estimates of mortality and morbidity among this subgroup of patients. Nursing professionals can also educate the significant others and the family regarding prehospital TBI guidelines.

The present study has demonstrated that there is a considerable scope of improvement in nurses' knowledge and this can be achieved in our setting through educational interventions. Ongoing training in the hospital area will increase the nurses' knowledge and awareness regarding TBI guidelines and care of TBI patients. Standardized trauma care protocols should be made by different institutions based on local realities and availability of resources. The national guidelines and consensus statement can be used for framing these protocols. As a first step of implementation, the effect of the guideline teaching for front-line employees like nurses can prove its practicality and usability. ${ }^{17}$

\section{Limitations}

Our study has certain limitations. The small sample selected through a purposive sampling technique and being limited to clinical nurses working in a single hospital makes it difficult to generalize the findings. In addition, gender differences could not be examined since the sample comprised only women. Further, the study focused only on the domain of knowledge.

Future studies should focus on the effect of teaching on the practice of the guidelines by the nurses and patient outcomes. Studies to assess the impact of teaching on improvement in patient outcomes are essential to evaluate the translation of evidence into practice. Hence, future studies can be planned in this aspect.

\section{Conclusion}

The existing knowledge gap about TBI guidelines among clinical nurses can be bridged effectively using educational interventions. Appropriately informed nurses can deliver adequate care and can contribute to improved patient outcomes. In line with these findings, there is an urgent need for nurse educators and administrators to create specific educational interventions for the nurses about TBI guidelines and thereby improve the nursing practice standards.

\section{Note}

Written informed consent was obtained from all individual participants included in the study. Anonymity and confidentiality were maintained throughout.

\section{Ethical Approval}

This study was approved by the institutional ethics committee. Formal administrative permission was obtained from medical superintendent.

\section{Funding}

None.

\section{Conflict of Interest}

None declared. 


\section{References}

1 Stocchetti N, Zanier ER. Chronic impact of traumatic brain injury on outcome and quality of life: a narrative review. Crit Care 2016;20(1):148

2 GBD 2016 Traumatic Brain Injury and Spinal Cord Injury Collaborators. Global, regional, and national burden of traumatic brain injury and spinal cord injury, 1990-2016: a systematic analysis for the Global Burden of Disease Study 2016. Lancet Neurol 2019;18(1):56-87

3 Maas AI. Traumatic brain injury in India: a big problem in need of data. Neurol India 2017;65(2):257-258

4 Gururaj G, Kolluri SV, Chandramouli BA, Subbakrishna DK, Kraus JF. Traumatic Brain Injury: National Institute of Mental Health \& NeuroSciences, Publication no.61, India;2005

5 Traumatic Brain Injury/ Indian Head Injury Foundation. (2015). Available from http://Indianhead injuryfoundation.org/traumatic-brain-injury/. Accessed March 15, 2021

6 Keris V, Lavendelis E, Macane I. Association between implementation of clinical practice guidelines and outcome for traumatic brain injury. World J Surg 2007;31(6):1352-1355

7 Bimal S, Kalia R, Singhi S, Kaur S. Development of Nursing Management Protocol for Pediatric Emergencies: Nursing and Midwifery Research Journal. 2009;5(2) available at: https:// docplayer.net/19465434-Development-ofnursing- management-protocol-for-p. Accessed March 15, 2021

8 Neurotrauma Society of India. Traumatic Brain Injury. Multi Organizational Consensus Recommendations for India. 2014. Available from http://ntsi.co.in/wp-content/uploads/2017/11/ Version.pdf. Accessed March 16, 2021

9 Kesinger MR, Nagy LR, Sequeira DJ, Charry JD, Puyana JC, Rubiano AM. A standardized trauma care protocol decreased in-hospital mortality of patients with severe traumatic brain injury at a teaching hospital in a middle-income country. Injury 2014;45(9):1350-1354

10 Herzer G, Illievich U, Voelckel WG, Trimmel H. Current practice in neurocritical care of patients with subarachnoid haemorrhage and severe traumatic brain injury: results of the Austrian Neurosurvey Study. Wein Kiln Wochenschr 2016;128(17-18):649-657

11 Shehab MS, Ibrahim NM, Elkadar HA. Impact of an Educational Program on Nurses' Knowledge and Practice Regarding Care of Traumatic Brain İnjury Patients at Intensive Care Unit at Suez Canal University Hospital. International Journal of Caring Sciences. 2018;11(2):1104

12 Oyesanya TO, Bowers BJ, Royer HR, Turkstra LS. Nurses' concerns about caring for patients with acute and chronic traumatic brain injury. J Clin Nurs 2018;27(7-8):1408-1419

13 Watts DD, Gibbons S, Kurzweil D. Mild traumatic brain injury: a survey of perceived knowledge and learning preferences of military and civilian nurses. J Neurosci Nurs 2011;43(3): 122-129, quiz 130-131

14 Ghoneim NIA, Alaa Elden SM, Okab ME, Elssay OEAE. Impact of implementing nursing care protocol on moderate head injured patient's outcome. J Am Sci 2012;8(7):649-664

15 Cook RS, Gillespie GL, Kronk R, et al. Effect of an educational intervention on nursing staff knowledge, confidence, and practice in the care of children with mild traumatic brain injury. J Neurosci Nurs 2013;45(2):108-118

16 Oyesanya TO, Snedden TR. Pediatric nurses' perceived knowledge and beliefs of evidence-based practice in the care of children and adolescents with moderate-to-severe traumatic brain injury. J Spec Pediatr Nurs 2018;23(2):e12209

17 Carney N, Totten AM, O'Reilly C, et al. Guidelines for the Management of Severe Traumatic Brain Injury, Fourth Edition. Neurosurgery 2017;80(1):6-15 